\title{
Prognostic impact of carbohydrate antigen 19-9 level at diagnosis in resected stage I-III pancreatic adenocarcinoma: a U.S. population study
}

\author{
Katelin A. Mirkin ${ }^{1}$, Christopher S. Hollenbeak ${ }^{1,2}$, Joyce Wong ${ }^{1}$ \\ ${ }^{1}$ Department of Surgery, ${ }^{2}$ Department of Public Health Sciences, The Pennsylvania State University, College of Medicine, Hershey, PA, USA \\ Contributions: (I) Conception and design: J Wong; (II) Administrative support: J Wong; (III) Provision of study materials or patients: CS Hollenbeak; \\ (IV) Collection and assembly of data: KA Mirkin, CS Hollenbeak; (V) Data analysis and interpretation: All authors; (VI) Manuscript writing: All \\ authors; (VII) Final approval of manuscript: All authors. \\ Correspondence to: Joyce Wong, MD. Department of Surgery, Division of Surgical Oncology, The Pennsylvania State University, College of Medicine, \\ 500 University Drive, MC H070, Hershey, PA 17033-0850, USA. Email: joyce.wong02@gmail.com.
}

\begin{abstract}
Background: Pancreatic adenocarcinoma is a highly aggressive cancer, with surgical resection and systemic therapy offering the only hope for long-term survival. Carbohydrate antigen 19-9 (CA 19-9) has been used as a prognostic marker after resection; however, the relationship between survival and pre-treatment CA 19-9 level remains unclear. This study evaluates pre-treatment serum CA 19-9 level as a predictor for long-term survival.
\end{abstract}

Methods: The U.S. National Cancer Data Base [2004-2012] was reviewed for patients with clinical stages I-III resected pancreatic adenocarcinoma with recorded pre-treatment CA 19-9 levels (U/mL). Kaplan Meier and Weibull survival analyses were performed.

Results: Four thousand seven hundred and one patients were included: $12.6 \%$ received neoadjuvant therapy (NAT), 27.4\% underwent surgery, and 60.1\% underwent surgery and adjuvant therapy. Amongst those who underwent initial surgery, there was no association between CA 19-9 levels $\leq 800(\leq 100,101-300$, 301-500, 501-800) with survival (stage I $\mathrm{P}=0.7592$, stage II $\mathrm{P}=0.5088$, stage III $\mathrm{P}=0.9037$ ). Levels $>800$ were associated with significantly worse survival in all stages $(\mathrm{P} \leq 0.0001$, all). Amongst those who received NAT, levels $>800$ were associated with worse survival in early (stage $\mathrm{I} P=0.0001$ ), but not advanced stage disease (stage II $\mathrm{P}=0.1891$, stage III $\mathrm{P}=0.9316$ ). In multivariable analyses, levels $>800$ demonstrated a 3.29 greater hazard of mortality with respect to patients with levels $\leq 100(\mathrm{P}<0.0001)$.

Conclusions: Pre-treatment CA 19-9 levels >800 appear to be associated with advanced disease, and are negatively associated with long-term survival. However, levels $\leq 800$ had no significant association with survival. Although this study suggests an association, further study is needed to evaluate whether patients with CA 19-9 levels >800 benefit from NAT.

Keywords: Pancreatic cancer; carbohydrate antigen 19-9 (CA 19-9)

Submitted Apr 14, 2017. Accepted for publication Jun 19, 2017.

doi: 10.21037/jgo.2017.07.04

View this article at: http://dx.doi.org/10.21037/jgo.2017.07.04

\section{Introduction}

Pancreatic adenocarcinoma is a highly aggressive cancer, with surgical resection and systemic therapy offering the only hope for long-term survival. Due to its anatomical location, the majority of patients present with advanced disease, with only $15-20 \%$ presenting as surgical candidates (1). Thus, there is a tremendous demand for prognostic markers.

One such marker is carbohydrate antigen 19-9 (CA 19-9), a sialylated Lewis blood group antigen regarded to be the most sensitive and specific serum marker for pancreatic 
cancer to date (2-4). While a CA 19-9 level of $37 \mathrm{U} / \mathrm{mL}$ is regarded as normal, a wide array of non-malignant elevators make the tumor marker a poor screening test in asymptomatic patients $(5,6)$. CA $19-9$ has been used as a prognostic marker after resection to monitor for recurrence and as a prognostic factor in inoperable cancer $(7,8)$. However, correlation of CA 19-9 level at diagnosis with survival in resectable pancreatic cancer remains unclear. This study evaluates serum CA 19-9 level prior to treatment as a predictor for long-term survival.

\section{Methods}

\section{Data}

The National Cancer Data Base (NCDB) is a clinical oncology database based on hospital registry data in the United States. The data reflects over $70 \%$ of newly diagnosed cancer cases [1998-2012] from over 1,500 Commission on Cancer accredited facilities. This was a retrospective cohort study of clinical data from this registry from 2004-2012. The study was deemed exempt by the M. S. Hershey Medical Center Human Subject Protection Board and it conforms to the provisions of in accordance with the Helsinski Declaration as revised in 2013, available at: http://www.wma.net/en/30publications/10policies/ b3/\%20index.html.

\section{Patient selection}

The NCDB was reviewed for patients diagnosed with clinical stages I-III resected pancreatic adenocarcinoma with CA 19-9 levels prior to treatment recorded. Patients with clinical stage IV disease or unknown stage were excluded. Clinical stage is a coded variable within the NCDB which is determined per individual institution. Histologies included in this analysis were carcinoma and adenocarcinoma identified using the third edition of the International Classification of Diseases for Oncology (ICD-O-3) primary site codes: C250-C254, C257-C259 and histology ICD-O-3 codes: 8050/3 8140/3, 8144/3, 8148/3, 8160/3, $8230 / 3,8255 / 3,2890 / 3,8310 / 3,8323 / 3,8342 / 3,8346 / 3$, $8380 / 3,8430 / 3,8460 / 3,8461 / 3,8480 / 3,8481 / 3,8500 / 3$, $8503 / 3,8504 / 3,8507 / 3,8510 / 3,8521 / 3,8523 / 3,8550 / 3$, $8560 / 3,8562 / 3,8570 / 3,8574 / 3$, and 8576/3. Patients who underwent an unknown surgical procedure, or local excision of tumor were excluded, in order to best capture those patients undergoing formal pancreatic resection. Patients with missing or unknown CA 19-9 levels were excluded. In the NCDB, CA 19-9 level is coded as 0-980 U/mL, with values over $980 \mathrm{U} / \mathrm{mL}$ coded simply as $\geq 980 \mathrm{U} / \mathrm{mL}$.

\section{Outcomes and covariates}

The primary outcome assessed was survival from diagnosis. The highest CA 19-9 lab value documented in the medical record prior to treatment was coded with the database. In order to best capture patients with malignancy related elevations in CA 19-9, the study population was stratified at a CA 19-9 level of $800 \mathrm{U} / \mathrm{mL}$. Covariates included sex, race, age, median income, insurance type and the Charlson/ Deyo comorbidity index (CCI). The CCI is an index of 15 common comorbidities including ranging from cardiac to pulmonary, to vascular, to renal, to endocrine disorders $(9,10)$.

Treatment facilities were characterized by geographic region and facility type. Disease was characterized by clinical and pathological variables including the American Joint Committee on Cancer (AJCC) clinical stage, number of lymph nodes sampled, number of positive lymph nodes, surgical margins, and pathological stage. Treatment was characterized by surgery type (distal pancreatectomy, whipple, total pancreatectomy and other), neoadjuvant treatment (NAT), and adjuvant treatment.

\section{Statistical analysis}

STATA software (version 12.1, StataCorp., College Station, TX, USA) was used to perform statistical analyses. Patient, disease, and treatment characteristics were compared between groups using student's $t$-tests, and chi square tests, as appropriate. Survival was assessed with Kaplan-Meier analyses stratified by CA 19-9 categories and a Weibull model.

\section{Results}

Of the 4,701 patients with clinically staged I-III resected pancreatic adenocarcinoma, $592(12.6 \%)$ received neoadjuvant therapy, 1,286 (27.4\%) underwent surgical resection alone, and 2,823 (60.1\%) underwent surgery followed by adjuvant therapy. The median CA 19-9 level was $888 \mathrm{U} / \mathrm{mL}$ (range, $0-980 \mathrm{U} / \mathrm{mL}$ ), with a distribution demonstrating $19.9 \%$ with CA $19-9$ levels $\leq 100 \mathrm{U} / \mathrm{mL}$, $12.2 \%$ between 100 and $300 \mathrm{U} / \mathrm{mL}, 6.6 \%$ between 300 and $500 \mathrm{U} / \mathrm{mL}, 7.1 \%$ between 500 and $800 \mathrm{U} / \mathrm{mL}$, and $54.2 \%$ $>800 \mathrm{U} / \mathrm{mL}$ (Figure 1).

Patient demographics stratified by treatment and 


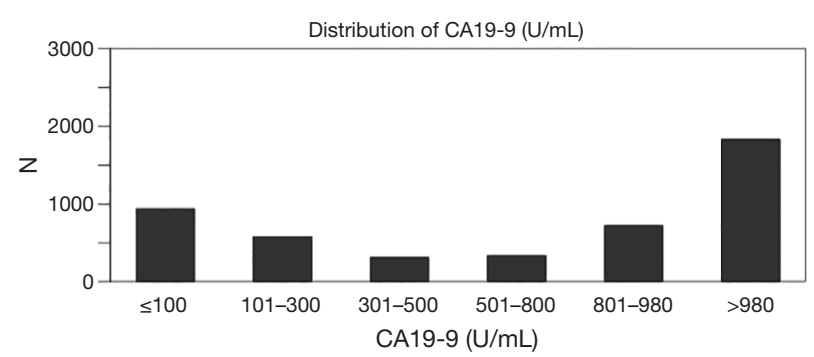

Figure 1 Distribution of CA 19-9 levels. CA 19-9, carbohydrate antigen 19-9.

CA 19-9 levels $\leq 800$ and $>800 \mathrm{U} / \mathrm{mL}$ are reported in Table 1 . Within the cohort of patients who underwent surgical resection alone or received adjuvant therapy, patients with CA 19-9 levels $\leq 800 \mathrm{U} / \mathrm{mL}$ were younger (66.0 vs. 66.8, $\mathrm{P}=0.0174)$, and non-white ( $23.4 \%$ vs. $21.3 \%, \mathrm{P}=0.002)$ with respect to patients with CA $19-9$ levels $\leq 800 \mathrm{U} / \mathrm{mL}$. Within the cohort of patients who received neoadjuvant therapy, patients with CA 19-9 levels $\leq 800 \mathrm{U} / \mathrm{mL}$ tended to be non-white $(25.0 \%$ vs. $14.3 \%, \mathrm{P}=0.007)$, but there was no difference in age $(\mathrm{P}=0.326)$ or sex $(\mathrm{P}=0.409)$.

Disease and treatment characteristics within the two cohorts are presented in Table 2. Among patients who underwent surgery with or without adjuvant therapy, those with CA 19-9 levels $>800 \mathrm{U} / \mathrm{mL}$ tended to have higher clinically staged disease $(\mathrm{P}=0.001)$ and pathologically staged disease $(\mathrm{P}<0.0001)$ relative to patients with $\mathrm{CA} 19-9$ levels $\leq 800 \mathrm{U} / \mathrm{mL}$. They were more likely to undergo a pancreaticoduodenectomy $(\mathrm{P}<0.001)$, have positive surgical margins $(\mathrm{P}<0.0001)$, greater numbers of positive regional lymph nodes $(\mathrm{P}<0.0001)$, and were less likely to receive adjuvant therapy $(\mathrm{P}<0.0001)$. Among patients who received neoadjuvant therapy, there was no statistically significant difference in clinical or pathological stage, surgical margins, or positive number of lymph nodes between patients with CA 19-9 levels $\leq 800 \mathrm{U} / \mathrm{mL}$ and $>800 \mathrm{U} / \mathrm{mL}$.

Kaplan-Meier survival analyses for patients who underwent surgical resection with or without adjuvant therapy are presented in Figure 2. There was no association of CA 19-9 levels $\leq 800 \mathrm{U} / \mathrm{mL}$ with survival at any stage (stage I $\mathrm{P}=0.7592$, stage II $\mathrm{P}=0.5088$, stage III $\mathrm{P}=0.9037$ ). CA 19-9 levels $>800 \mathrm{U} / \mathrm{mL}$ were associated with significantly worse survival in all clinical stages of disease (stage I $\mathrm{P}<0.0001$, stage II $\mathrm{P}<0.0001$, stage III $\mathrm{P}<0.0001$ ) (Figure 3).

Kaplan-Meier survival analyses for patients who received neoadjuvant therapy followed by surgical resection are presented in Figure 4. There was no association of CA
19-9 levels $\leq 800 \mathrm{U} / \mathrm{mL}$ with survival in early stage disease (stage I $\mathrm{P}=0.3030$, stage II $\mathrm{P}=0.2908$ ). While there was a statistically significant difference in survival among CA 19-9 levels below 800 in stage III disease $(\mathrm{P}=0.0464)$, this analysis was limited by small sample size $(\mathrm{n}=35)$. CA 19-9 levels $>800 \mathrm{U} / \mathrm{mL}$ were associated with significantly worse survival in stage I disease $(\mathrm{P}=0.0001)$, but not in stage II or III disease ( $\mathrm{P}=0.2029, \mathrm{P}=0.9215$, respectively) (Figure 5).

After controlling for patient, disease, and treatment characteristics, patients who received neoadjuvant or adjuvant therapy had a lower hazard of mortality relative to patients who underwent surgical resection alone (HR 0.60, $\mathrm{P}<0.001$, HR 0.52, $\mathrm{P}<0.001$ ) (Table 3). Patients with CA 19-9 levels $801-980 \mathrm{U} / \mathrm{mL}$ or $>980 \mathrm{U} / \mathrm{mL}$ had significantly greater hazards of mortality relative to patients with CA 19-9 levels $\leq 100 \mathrm{U} / \mathrm{mL}$ (HR 3.29, $\mathrm{P}<0.001$, HR 1.51, $\mathrm{P}<0.001$, respectively).

\section{Discussion}

While a CA 19-9 level of $37 \mathrm{U} / \mathrm{mL}$ is widely regarded as normal, there are varying reports of levels associated with malignancy prognosis (5). This study found an association between pre-treatment CA 19-9 levels $>800 \mathrm{U} / \mathrm{mL}$ and advanced stage disease. A CA 19-9 level $>800 \mathrm{U} / \mathrm{mL}$ was associated with worse survival in all clinical stages of disease, and was found to be an independent poor prognostic factor. Some have used CA 19-9 levels to characterize resectability. A study of 244 patients with pancreatic adenocarcinoma, reported a $70 \% \mathrm{R} 0$ resection in patients with both negative preoperative CA 19-9 and CEA levels (11). A number of small single institution studies have evaluated the predictive value of CA 19-9 levels in resectability. The CA 19-9 level reported as predictive of resectability has ranged from 92.8 to $622 \mathrm{U} / \mathrm{mL}(11-14)$. To our knowledge, this is the first study to evaluate the prognostic impact of a wide range of CA 19-9 levels.

In this study, neoadjuvant therapy was associated with a survival benefit in patients with CA 19-9 levels $>800 \mathrm{U} / \mathrm{mL}$. A broad study by Bergquist et al. evaluating patients with stage I and II disease, reported that though elevations in CA 19-9 levels $>37 \mathrm{U} / \mathrm{mL}$ were associated with poorer survival, administration of neoadjuvant chemotherapy mitigated this effect (15). While surgery and systemic adjuvant therapy are standard of care, as few as half may actually receive intended adjuvant therapy $(16,17)$. In this study, nearly one third underwent surgical resection, but received no form of systemic therapy. Receipt of neoadjuvant therapy has been 
Table 1 Patient demographics

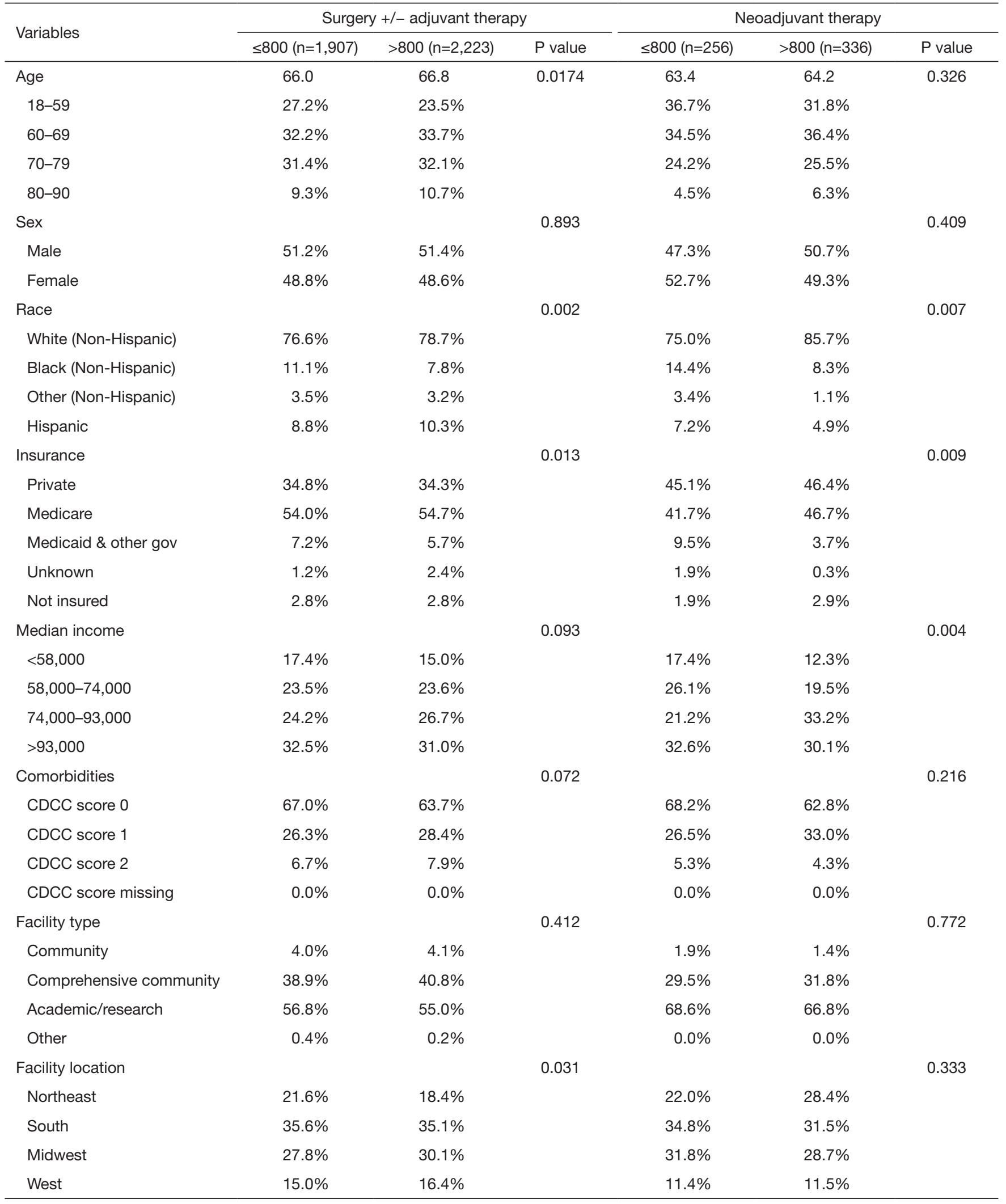


Table 2 Disease and treatment characteristics

\begin{tabular}{|c|c|c|c|c|c|c|}
\hline Variables & \multicolumn{3}{|c|}{ Surgery $+/-$ adjuvant therapy } & \multicolumn{3}{|c|}{ Neoadjuvant therapy } \\
\hline Clinical stage & & & 0.001 & & & 0.059 \\
\hline Stage I & $44.8 \%$ & $40.5 \%$ & & $27.7 \%$ & $22.6 \%$ & \\
\hline Stage II & $51.5 \%$ & $55.0 \%$ & & $59.1 \%$ & $57.3 \%$ & \\
\hline Surgery type & & & $<0.0001$ & & & 0.682 \\
\hline Distal resection & $16.2 \%$ & $11.6 \%$ & & $11.4 \%$ & $8.9 \%$ & \\
\hline Whipple & $51.0 \%$ & $54.8 \%$ & & $53.8 \%$ & $55.6 \%$ & \\
\hline Total resection & $12.5 \%$ & $12.2 \%$ & & $14.4 \%$ & $16.3 \%$ & \\
\hline Positive regional lymph nodes & 2.2 & 3.0 & $<0.0001$ & 1.2 & 1.1 & 0.754 \\
\hline Surgical margins & & & $<0.0001$ & & & 0.855 \\
\hline No residual tumor & $76.0 \%$ & $70.0 \%$ & & $81.1 \%$ & $78.5 \%$ & \\
\hline Residual tumor, NOS & $8.4 \%$ & $9.5 \%$ & & $8.0 \%$ & $7.7 \%$ & \\
\hline Microscopic residual tumor & $12.5 \%$ & $15.4 \%$ & & $7.2 \%$ & $8.6 \%$ & \\
\hline Macroscopic residual tumor & $0.9 \%$ & $1.2 \%$ & & $0.4 \%$ & $0.9 \%$ & \\
\hline Indeterminate or unknown & $2.3 \%$ & $4.0 \%$ & & $3.4 \%$ & $4.3 \%$ & \\
\hline Pathological stage & & & $<0.0001$ & & & 0.058 \\
\hline Unknown & $5.2 \%$ & $7.2 \%$ & & $9.1 \%$ & $12.6 \%$ & \\
\hline Treatment & & & $<0.0001$ & & & 1.000 \\
\hline Any NAT & $0.0 \%$ & $0.0 \%$ & & $100.0 \%$ & $100.0 \%$ & \\
\hline Surgery only & $26.9 \%$ & $35.1 \%$ & & $0.0 \%$ & $0.0 \%$ & \\
\hline Any adj & $73.1 \%$ & $64.9 \%$ & & $0.0 \%$ & $0.0 \%$ & \\
\hline
\end{tabular}

NOS, not otherwise specified; NAT, neoadjuvant therapy; adj, adjuvant therapy.

associated with survival benefits in advanced pancreatic cancer, and appears to offer survival benefits in patients with elevated CA 19-9 $(18,19)$. Elevations in the biomarker, particularly $>800 \mathrm{U} / \mathrm{mL}$ should prompt clinicians to consider neoadjuvant therapy.

To our knowledge, this is the largest and most contemporary analysis of CA 19-9 levels at diagnosis of pancreatic adenocarcinoma, and the only to examine the impact of CA 19-9 elevations at multiple levels in resectable pancreatic cancer. However, there are some important limitations which should be acknowledged, including those inherent to retrospective reviews of large databases. 

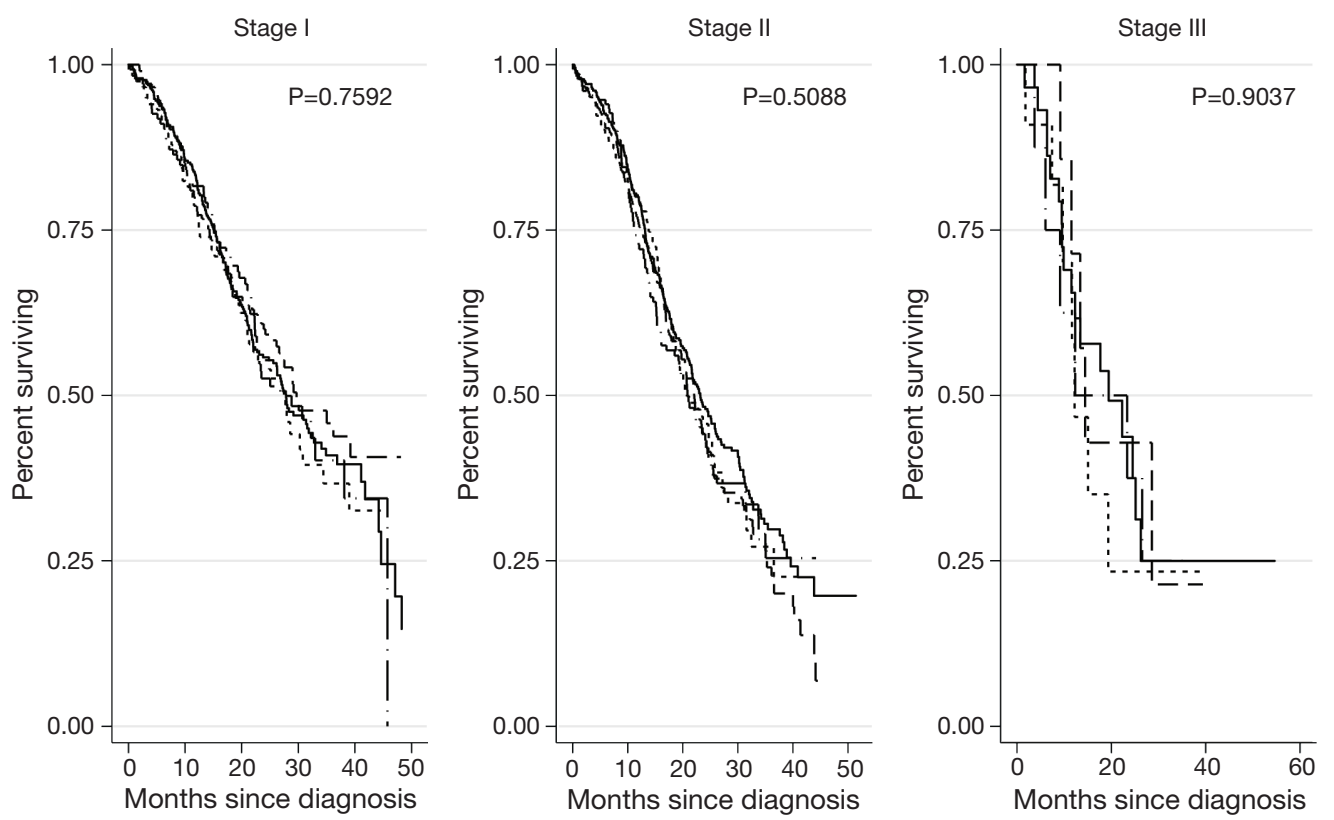

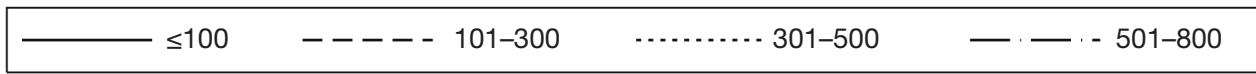

Figure 2 Kaplan Meier analyses for patients who underwent surgery +/- adjuvant therapy by CA 19-9 level (U/mL). CA 19-9, carbohydrate antigen 19-9.
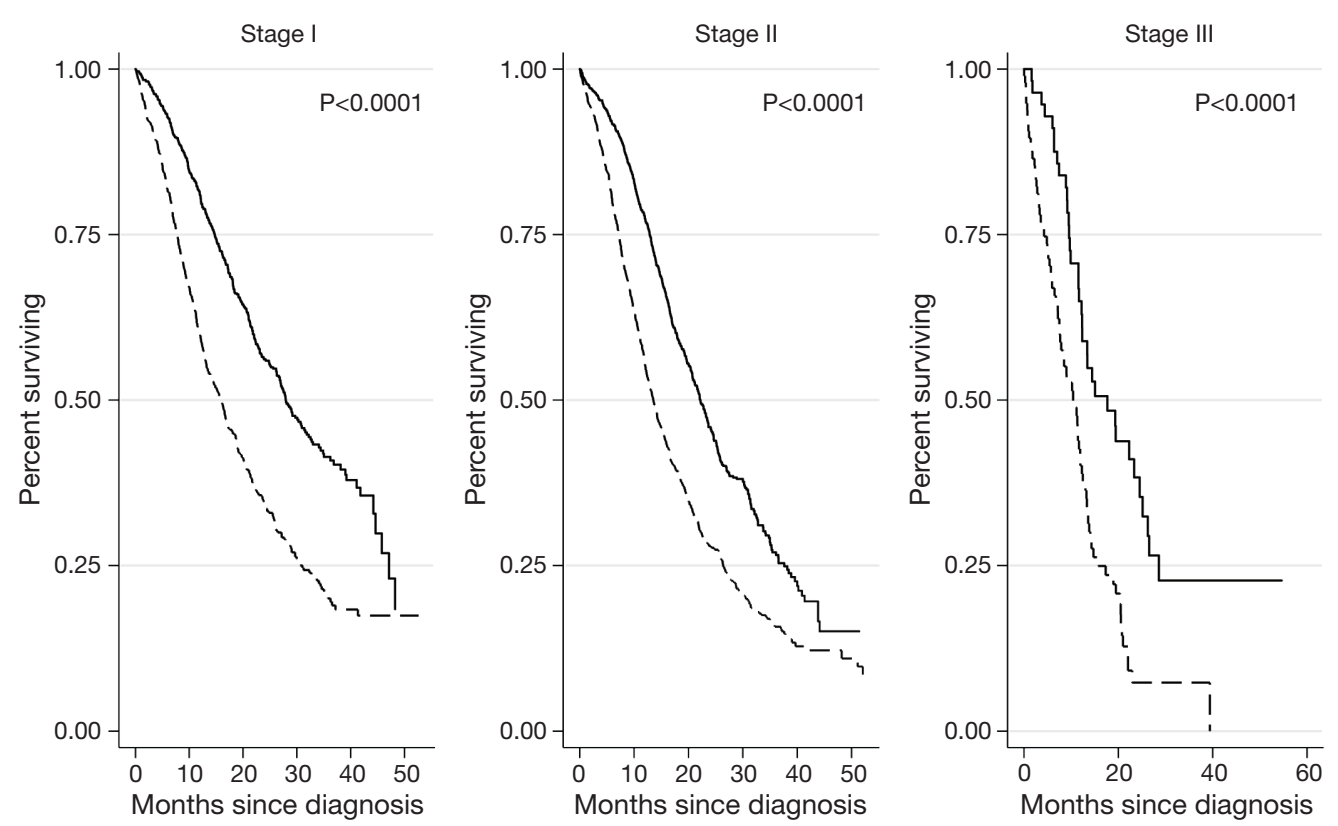

CA19-9 $\leq 800 \mathrm{U} / \mathrm{mL}$ ----- CA19-9 $>800 \mathrm{U} / \mathrm{mL}$

Figure 3 Kaplan Meier analyses for patients who underwent surgery +/- adjuvant therapy by CA 19-9 level (U/mL). CA 19-9, carbohydrate antigen 19-9. 

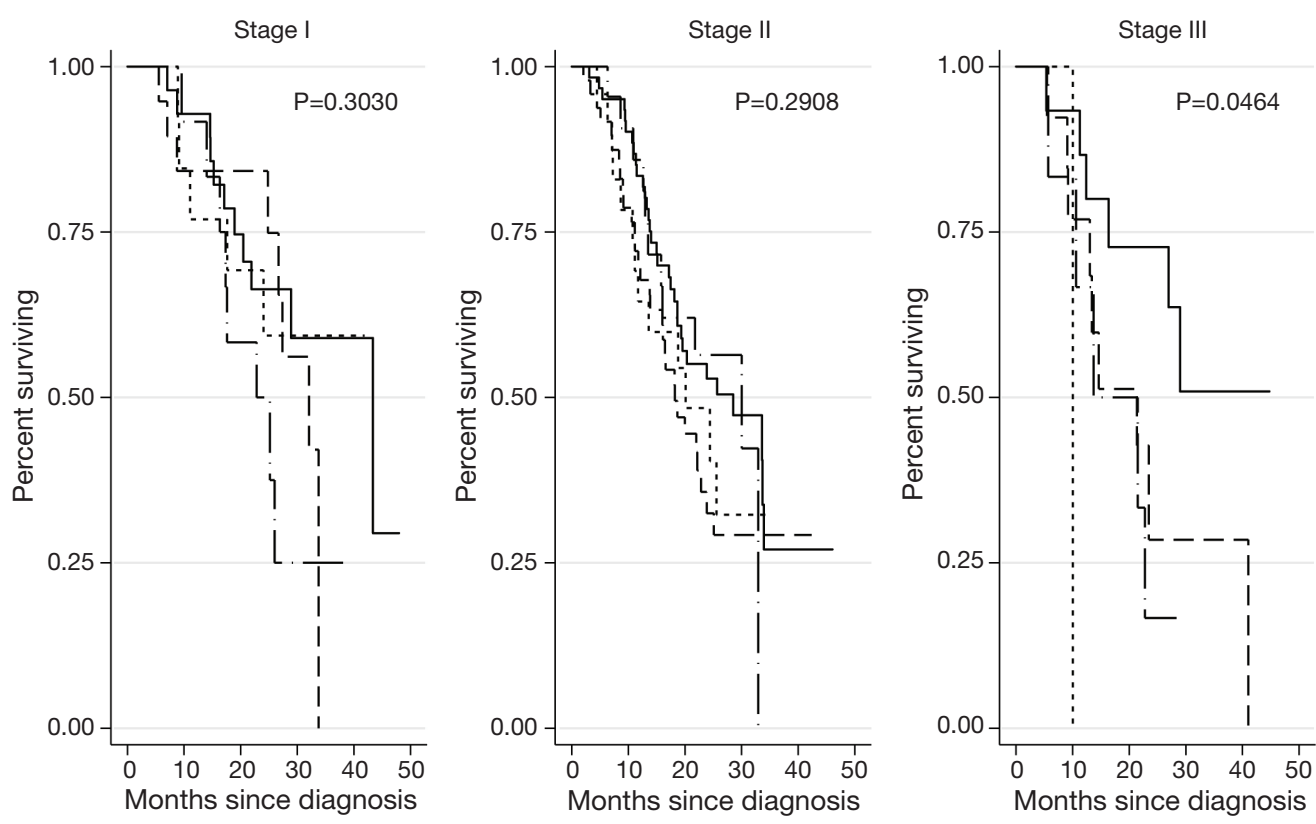

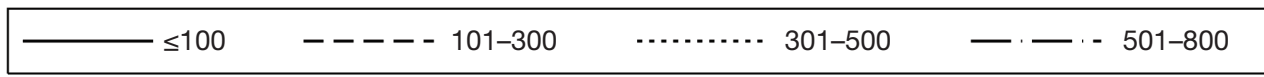

Figure 4 Kaplan Meier analyses for patients who received neoadjuvant therapy followed by surgery, by CA 19-9 level (U/mL). CA 19-9, carbohydrate antigen 19-9.
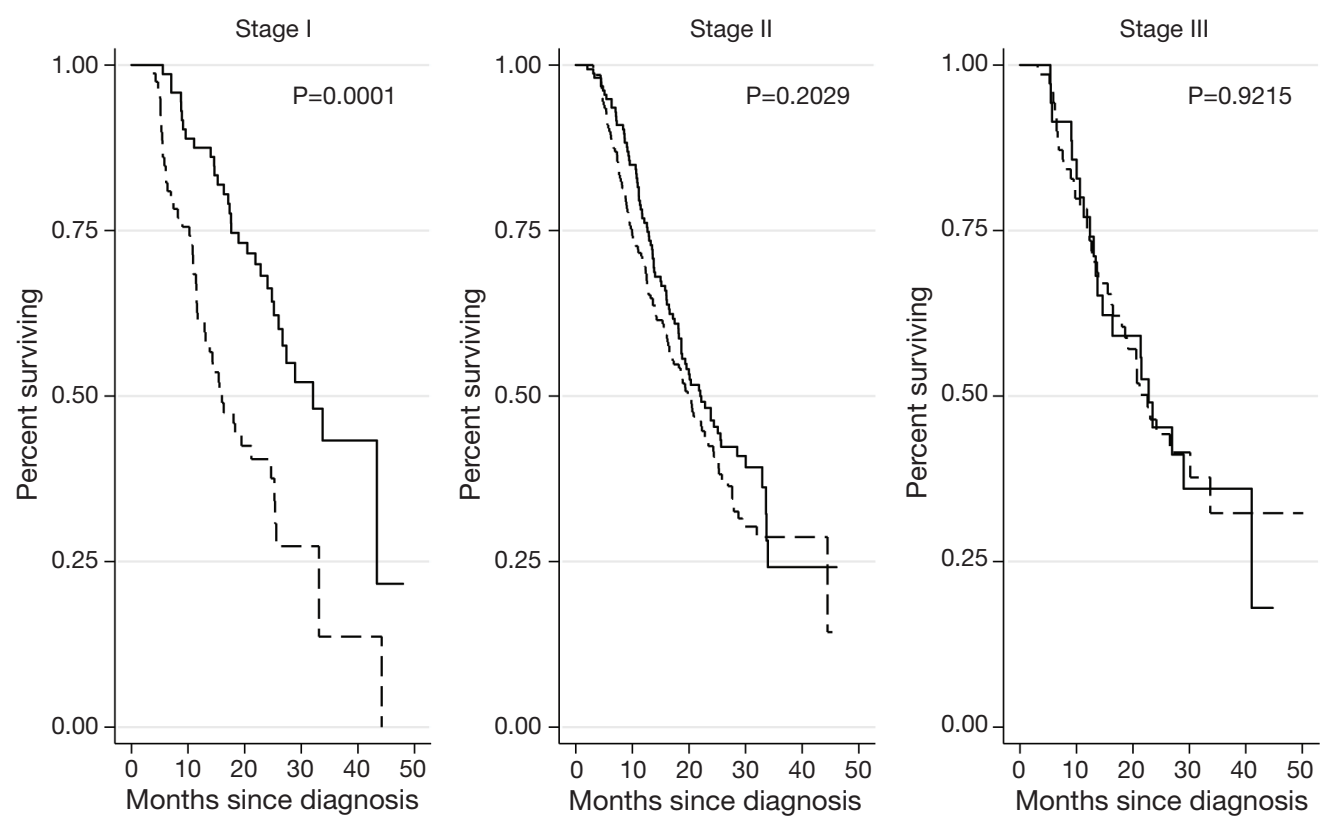

$\mathrm{CA} 19-9 \leq 800 \mathrm{U} / \mathrm{mL}$

----- CA19-9 $>800 \mathrm{U} / \mathrm{mL}$

Figure 5 Kaplan Meier analyses for patients who received neoadjuvant therapy followed by surgery, by CA 19-9 level (U/mL). CA 19-9, carbohydrate antigen 19-9. 
Table 3 Multivariate survival analysis

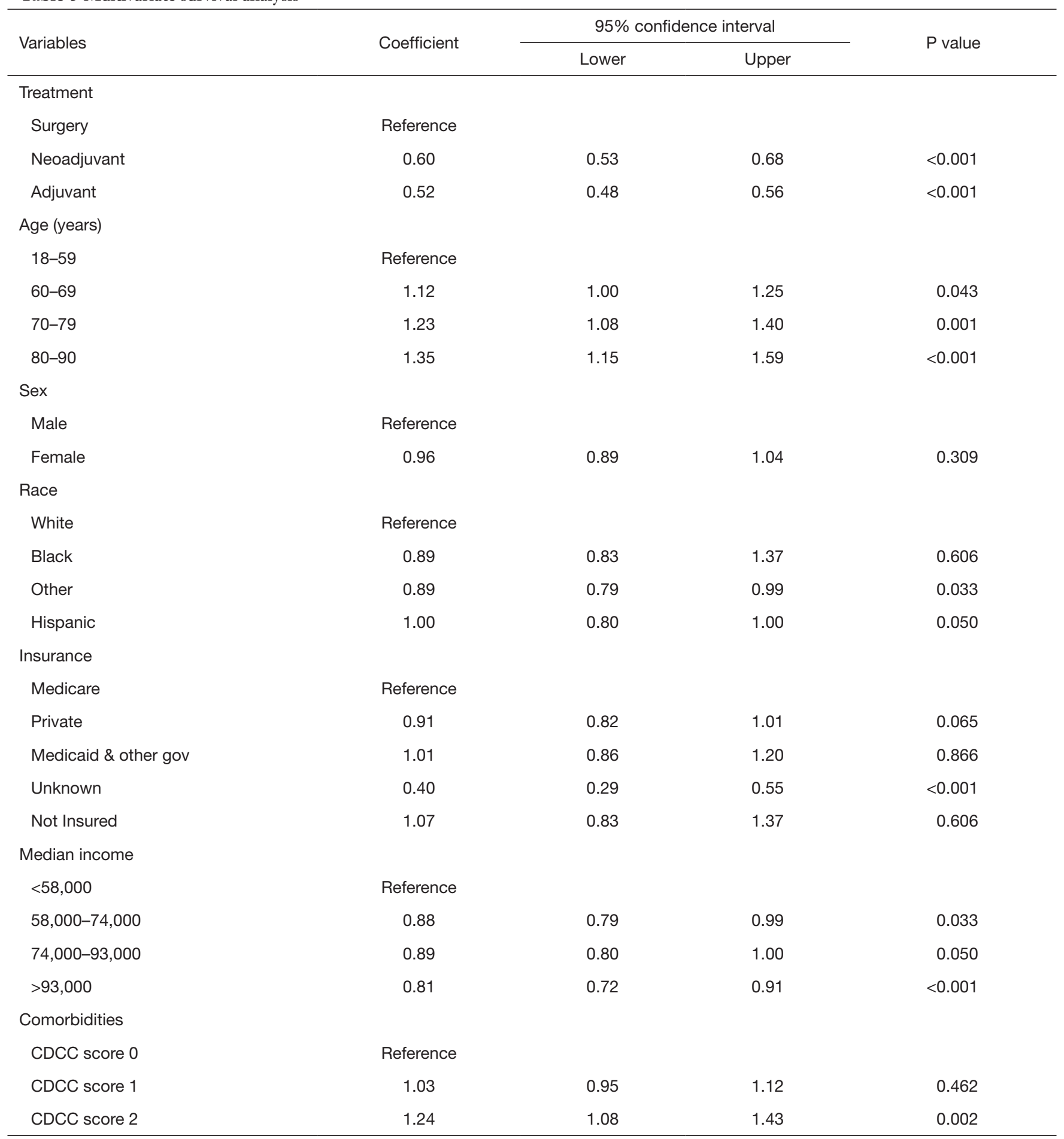

Table 3 (continued) 
Table 3 (continued)

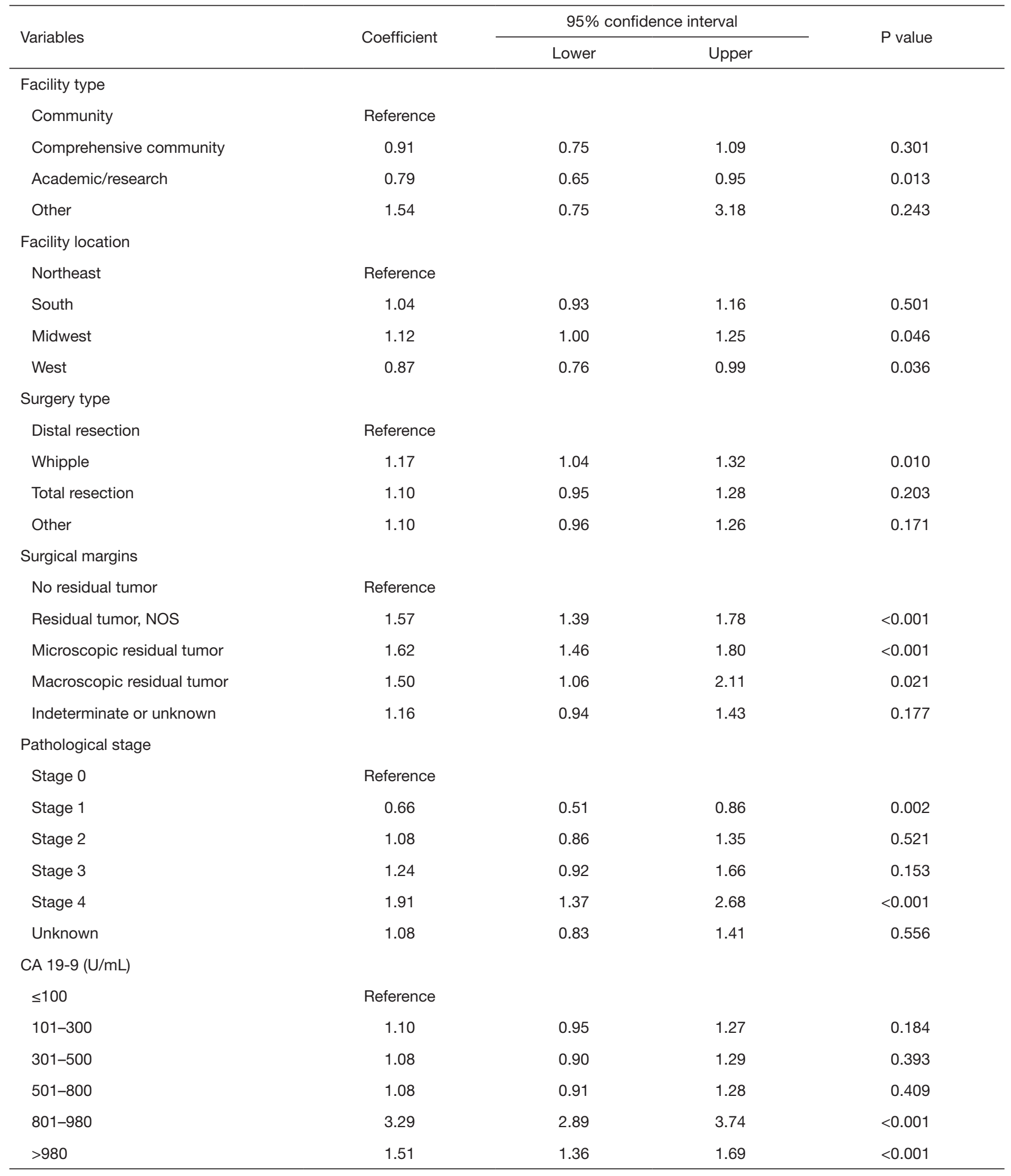

NOS, not otherwise specified. 
The NCDB codes only for the highest CA 19-9 value documented in the medical record prior to treatment. These values are derived from a heterogenous population of hospitals with non-uniform biomarker assays. Additionally, the NCDB does not code for potential confounders of CA 19-9 elevation such as PPI use, or biliary obstruction. Given the retrospective nature of the study, the treatment cohorts likely contain a selection bias.

\section{Conclusions}

An elevated CA 19-9 level $>800 \mathrm{U} / \mathrm{mL}$ appears to be associated with more advanced stage disease; however, CA 19-9 $\leq 800$ drawn prior to treatment had no significant association with long-term survival. CA 19-9 levels $>800 \mathrm{U} / \mathrm{mL}$ were negatively associated with long-term survival. Although this study suggests an association, further study is needed to evaluate whether patients with CA 19-9 levels > $800 \mathrm{U} / \mathrm{mL}$ benefit from NAT.

\section{Acknowledgements}

None.

\section{Footnote}

Conflicts of Interest: This study was selected and presented as a poster presentation at the European Society of Medical Oncology 18th World Congress on Gastrointestinal Cancer, June 29-July 2, 2016, Barcelona, Spain.

Ethical Statement: The study was deemed exempt by the M. S. Hershey Medical Center Human Subject Protection Board and it conforms to the provisions of in accordance with the Helsinski Declaration as revised in 2013, available at: http://www.wma.net/en/30publications/10policies/ b3/\%20index.html.

Disclaimer: The National Cancer Data Base (NCDB) is a joint project of the Commission on Cancer $(\mathrm{CoC})$ of the American College of Surgeons and the American Cancer Society. The CoC's NCDB and the hospitals participating in the $\mathrm{CoC} \mathrm{NCDB}$ are the source of the de-identified data used herein; they have not verified and are not responsible for the statistical validity of the data analysis or the conclusions derived by the authors.

\section{References}

1. Bilimoria KY, Bentrem DJ, Ko CY, et al. Validation of the 6th edition AJCC Pancreatic Cancer Staging System: report from the National Cancer Database. Cancer 2007;110:738-44.

2. Pleskow DK, Berger HJ, Gyves J, et al. Evaluation of a serologic marker, CA 19-9, in the diagnosis of pancreatic cancer. Ann Intern Med 1989;110:704-9.

3. Cwik G, Wallner G, Skoczylas T, et al. Cancer antigens $19-9$ and 125 in the differential diagnosis of pancreatic mass lesions. Arch Surg 2006;141:968-73.

4. van den Bosch RP, van Eijck CH, Mulder PG, et al. Serum CA19-9 determination in the management of pancreatic cancer. Hepatogastroenterology 1996;43:710-3.

5. Kim JE, Lee KT, Lee JK, et al. Clinical usefulness of carbohydrate antigen 19-9 as a screening test for pancreatic cancer in an asymptomatic population. J Gastroenterol Hepatol 2004;19:182-6.

6. Chang CY, Huang SP, Chiu HM, et al. Low efficacy of serum levels of CA 19-9 in prediction of malignant diseases in asymptomatic population in Taiwan. Hepatogastroenterology 2006;53:1-4.

7. Maisey NR, Norman AR, Hill A, et al. CA19-9 as a prognostic factor in inoperable pancreatic cancer: the implication for clinical trials. Br J Cancer 2005;93:740-3.

8. Berger AC, Garcia M Jr., Hoffman JP, et al. Postresection CA 19-9 predicts overall survival in patients with pancreatic cancer treated with adjuvant chemoradiation: a prospective validation by RTOG 9704. J Clin Oncol 2008;26:5918-22.

9. Deyo RA, Cherkin DC, Ciol MA. Adapting a clinical comorbidity index for use with ICD-9-CM administrative databases. J Clin Epidemiol 1992;45:613-9.

10. Charlson ME, Pompei P, Ales KL, et al. A new method of classifying prognostic comorbidity in longitudinal studies: development and validation. J Chronic Dis 1987;40:373-83.

11. Fujioka S, Misawa T, Okamoto T, et al. Preoperative serum carcinoembryonic antigen and carbohydrate antigen 19-9 levels for the evaluation of curability and resectability in patients with pancreatic adenocarcinoma. J Hepatobiliary Pancreat Surg 2007;14:539-44.

12. Karachristos A, Scarmeas N, Hoffman JP. CA 19-9 levels predict results of staging laparoscopy in pancreatic cancer. J Gastrointest Surg 2005;9:1286-92.

13. Maithel SK, Maloney S, Winston C, et al. Preoperative CA 
19-9 and the yield of staging laparoscopy in patients with radiographically resectable pancreatic adenocarcinoma. Ann Surg Oncol 2008;15:3512-20.

14. Kiliç M, Göçmen E, Tez M, et al. Value of preoperative serum CA 19-9 levels in predicting resectability for pancreatic cancer. Can J Surg 2006;49:241-4.

15. Bergquist JR, Puig CA, Shubert CR, et al. Carbohydrate antigen 19-9 elevation in anatomically resectable, early stage pancreatic cancer is independently associated with decreased overall survival and an indication for neoadjuvant therapy: a national cancer database study. J Am Coll Surg 2016;223:52-65.

16. Oettle H, Neuhaus P, Hochhaus A, et al. Adjuvant chemotherapy with gemcitabine and long-term outcomes

Cite this article as: Mirkin KA, Hollenbeak CS, Wong J. Prognostic impact of carbohydrate antigen 19-9 level at diagnosis in resected stage I-III pancreatic adenocarcinoma: a U.S. population study. J Thorac Dis 2017;8(5):778-788. doi: 10.21037/jgo.2017.07.04 among patients with resected pancreatic cancer: the CONKO-001 randomized trial. JAMA 2013;310:1473-81.

17. Bilimoria KY, Bentrem DJ, Ko CY, et al. Multimodality therapy for pancreatic cancer in the U.S. : utilization, outcomes, and the effect of hospital volume. Cancer 2007;110:1227-34.

18. Mirkin KA, Hollenbeak CS, Wong J. Survival impact of neoadjuvant therapy in resected pancreatic cancer: a prospective cohort study involving 18,332 patients from the national cancer data base. Int J Surg 2016;34:96-102.

19. Gillen S, Schuster T, Meyer Zum Buschenfelde C, et al. Preoperative/neoadjuvant therapy in pancreatic cancer: a systematic review and meta-analysis of response and resection percentages. PLoS Med 2010;7:e1000267. 